\title{
OBJECTIVE EVALUATION OF PATIENT-VENTILATOR INTERACTIONS DURING NONINVASIVE VENTILATION (NIV)
}

Herinaina Rabarimanantsoa*, Linda Achour, PhD", , Christophe Letellier, PhD*, Jean-Francois Muir, MD, PhD", and Antoine Cuvelier, MD, PhD",

Noninvasive Ventilation Awards, sponsored by ResMed

*CORIA UMR 6614, University of Rouen

${ }^{*}$ ADIR Association, Hôpital de Bois guillaume, Rouen

"Pulmonary and Respiratory Intensive Care Department, Rouen University Hospital \& UPRES EA 3830 (IFR MP23), Institute for Biomedical Research, University of Rouen, France

WINNING ABSTRACT: The success of NIV depends on patient-ventilator interactions. These interactions are evaluated with the subjective comfort score which is not always reliable. An objective evaluation is thus required. To evaluate these interactions, we use a statistical measure of the variability of a physiological signal, i.e the Shannon entropy. Our purpose is to show whether estimating Shannon entropy from airway pressure (SP) and from the total duration of ventilatory cycles (ST) may evaluate objectively the patient-ventilator interactions during NIV.

Pressure support NIV was applied to 4 COPD patients, 4 OHS patients in stable state and 4 healthy subjects during six successive 10-min periods with various inspiratory pressure. The flow and the airway pressure signals were recorded with sensors located near the mask. Good patient-ventilator interactions were assumed to correspond to patient well synchronized (low ineffective efforts) and with low ventilatory variability. All the subjects were awaked and both Shannon entropies SP and ST were computed for each ventilatory tracing.

The incidence of ineffective efforts (IE) varied from 0 to $64.7 \%$. SP appeared to be strongly correlated to this incidence $(r=0.91)$. ST quantified precisely the ventilatory variability. When SP was plotted versus ST, 4 distinct groups of patients were distinguished as follows:

$\mathrm{SP}<1$ and $\mathrm{ST}<\mathbf{1}: \mathrm{IE}<\mathbf{1 0} \%$ but no ventilatory variability

$\mathrm{SP}<1$ and $\mathrm{ST}>1$ : IE $<10 \%$ with high ventilatory variability

SP $>1$ and $S T<1:$ IE $>10 \%$ but no ventilatory variability

SP $>1$ and $S T>1:$ IE $>10 \%$ with high ventilatory variability

Shannon entropies objectively evaluate the patient-ventilator interactions in terms of ineffective efforts and ventilatory variability. Good patient-ventilator interactions occur when there is only few ineffective efforts $\left(S_{P}<1\right)$ and a low ventilatory variability $(\mathrm{S} T<1)$.

\section{KEYWORDS: Chronic respiratory failure, noninvasive ventilation, patient-ventilator interactions, Shannon entropy}

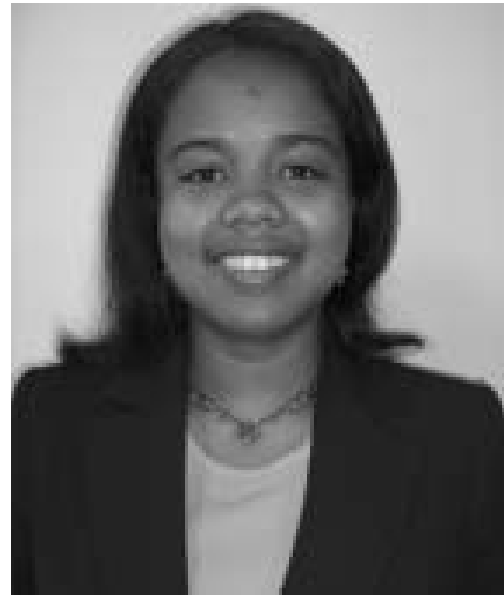

Herinaina Rabarimanantsoa

Research Laboratory CORIA UMR 6614 \& Rouen University Hospital, Rouen, France

STATEMENT OF INTEREST: None declared.

\section{MY JOB AND THE UNIT IN WHICH I WORK}

I am currently a PhD student in a physics laboratory (Complexe de Recherche Interprofessionnelle en Aérothermochimie CORIA) specialising in fluid mechanics, energetics and bioengineering at the University of Rouen, France. My PhD thesis is dedicated to patient-ventilator interactions during sleep. To that end, I am working with Professor Christophe Letellier, my thesis supervisor, who has been working in the area of nonlinear dynamic systems theory for a long time. He is particularly involved with the development of tools to characterise complex dynamics and to apply them to data from the real world, such as biomedical data [1, 2]. My work is also performed in collaboration with Professors Antoine Cuvelier and Jean-François Muir, who are both respiratory physicians involved daily with patients treated by noninvasive ventilation (NIV) at the Respiratory Intensive Care Unit of Rouen University Hospital. These physicians have been involved in several clinical works concerning the technical aspects, the monitoring and the assessment of clinical efficacy during NIV in chronic and acute respiratory failure. Moreover, this collaboration also involves Linda Achour, who is a biomedical research engineer at the Rouen University (UPRES EA IFRMP 23). She has developed a 
large expertise about lung test studies using noninvasive ventilators and demand oxygen delivery systems. I am now contributing to this expertise by developing tools using nonlinear dynamic systems theory and applying these tools to bench tests results and also to clinical data obtained from patients with respiratory failure.

\section{MY WINNING ABSTRACT AS PART OF MY RESEARCH/ MY RESEARCH AS PART OF MY WORKING GROUP}

NIV is now largely considered to be an efficient therapeutic option in patients suffering from chronic respiratory failure. This treatment is mostly prescribed to be used during sleep, but very few studies have analysed patient-ventilator interactions during sleep. One of the reasons for this is the very large number of ventilatory cycles (at least $>10,000$ ) that are recorded during a conventional 7-h night in a sleep laboratory and that cannot be manually analysed on a routine basis. Therefore, the impact of patient-ventilator interactions on sleep remains unknown. We developed an algorithm that can automatically provide the rate of ineffective triggering efforts (inspiratory efforts not followed by a ventilator triggering) during pressure-support NIV. This rate may be of clinical value since it may reduce the sleep efficacy and, subsequently, the patient's comfort [3].

Therefore, we developed the "Shannon entropy", a statistical measure of the variability of a physiological signal, in order to analyse the signals recorded during pressure-support NIV and to better assess patient-ventilator interactions. With this methodology, we obtained a qualitative and quantitative assessment of a good synchronisation based on a low rate of ineffective triggering efforts and a low ventilatory variability. In our study, we proved that two Shannon entropies applied to variables from ventilatory cycles (maxima of airway pressure and total duration of each ventilatory cycle) appeared to be correlated to the rate of ineffective triggering efforts and to the ventilatory variability [2]. Using the Shannon entropy avoids the manual counting of nontriggered cycles and allows an objective assessment of some patient-ventilator interactions. The next step will be to study Shannon entropies during longterm home mechanical ventilation. In our working group, other tools issued from chaos theory are being developed and applied to respiratory data obtained under NIV. Thus, physicians will have the opportunity to validate them in clinical practice [1] in order to understand why NIV sometimes fails or is badly tolerated.

\section{THE IMPACT OF MY WORK ON CLINICAL OR RESEARCH PRACTICE}

At the present time, setting a ventilator is mainly based on the experience of the team who initiate the treatment. The physicians are clearly aware of the importance of patientventilator interactions, including synchronisation and ventilatory variability. As our two Shannon entropies, from airway pressure (SP) and from the total duration of ventilatory cycles

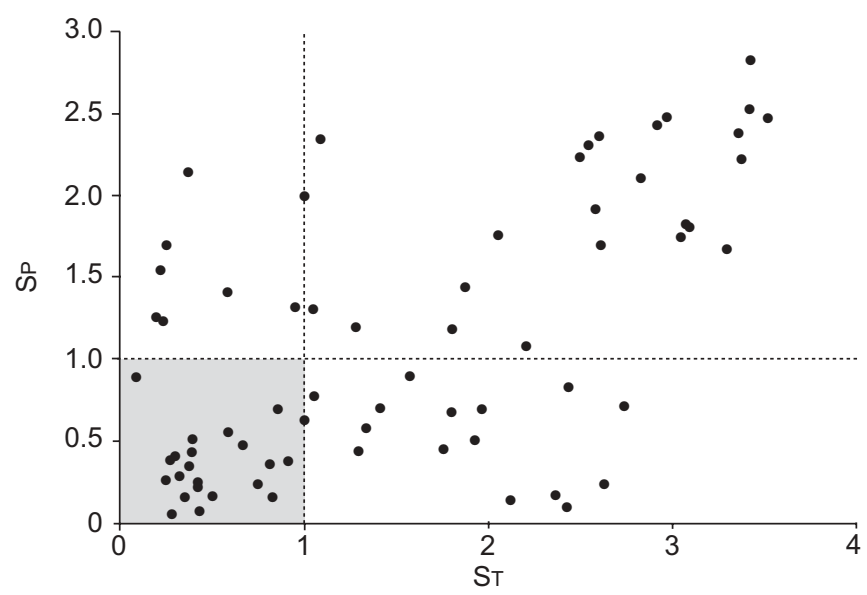

FIGURE 1. Graph showing the improvements of patient-ventilator interactions during noninvasive ventilation. ST: Shannon entropy from the total duration of ventilatory cycles (related to ventilatory variability); SP: Shannon entropy from the airway pressure (related to ineffective triggering efforts). $\bullet$ : each represents one patient; : : optimal interaction (where SP and ST $<1$ ).

(ST), quantify the rate of ineffective triggering efforts and the ventilatory variability during pressure support NIV, we developed a map (fig. 1) in order to follow the improvements of patient-ventilator interactions. The four distinct patterns defined by entropies equal to 1, allow us to distinguish four types of patient-ventilator interaction. The optimal interactions correspond to $\mathrm{SP}<1$ and $\mathrm{ST}<1$ : the optimal zone. Obviously, all the patients are not located in this optimal zone due to the lack of effective triggering and other psychological causes.

All Shannon entropy calculations may be integrated within the ventilator for clinical use at the patient's bedside.

\section{ACKNOWLEDGEMENTS}

I would like to thank Bernard Gasparutto, Dominique Metayer, Gilles Petit and François Dugardin (ADIR Assistance, member of the ANTADIR Federation) and ADIR Association for their invaluable help and constant support during my PhD thesis.

\section{REFERENCES}

1 Achour L, Letellier C, Cuvelier A, Verin E, Muir JF. Asynchrony and cyclic variability in pressure support noninvasive ventilation. Comput Biol Med 2007; 37: 1308-1320.

2 Rabarimanantsoa H, Achour L, Letellier C, Cuvelier A, Muir JF. Recurrence plots and Shannon entropy for a dynamical analysis of asynchronisms in noninvasive mechanical ventilation. Chaos 2007; 17: 013115.

3 Fanfulla F, Delmastro M, Berardinelli A, Lupo ND, Nava S. Effects of different ventilator settings on sleep and inspiratory effort in patients with neuromuscular disease. Am J Resp Crit Care Med 2005; 172: 619-624. 